A C T A C H E M I A S C A N D I N A V I C A 19 (1965) $401-413$

\title{
Semi-empirical Molecular Orbital Studies of Aromatic Hydrocarbons. II
}

\author{
P. N. SKA N C K E
}

Institute for Theoretical Physics, Vanadisvägen 9, Stockholm Va, Sweden

\begin{abstract}
The electronic structures and electronic spectra of the molecules phenanthrene, chrysene, and perylene have been studied within the Pariser-Parr-Pople approximation. The molecular ground states are described by a single determinant wave function, whereas configuration interaction, including all the singly excited configurations, has been invoked in the description of the electronically excited states. Special attention has been paid to the abnormally long carboncarbon bonds observed in these molecules, and in the case of perylene two different molecular models have been used in the evaluation of the semi-empirical parameter values. Predicted bond distances, ionization potentials, and electronic transitions are discussed in comparison with experimental observations.
\end{abstract}

\section{INTRODUCTION}

$\mathrm{T}_{\mathrm{r}}$ he carbon-carbon bond distances in aromatic hydrocarbons are usually found to be about $1.40 \AA$. The small fluctuations of individual bond distances around this value are rather well accounted for in terms of $\pi$-electron distributions resulting from calculations within the Hückel approximation.

In some polynuclear aromatic hydrocarbons, however, the presence of carbon-carbon bond distances exceeding the normal aromatic bond length by substantial amounts has been firmly established. A typical example is the length of the peri-bonds in perylene for which values of $1.515 \AA^{1}$ and $1.471 \AA^{2}$ recently have been reported. By an accurate $\mathrm{X}$-ray crystallographic study ${ }^{3}$ of chrysene a value of $1.468 \AA$ for one of the bond distances has been found. Also in phenanthrene an unusually long bond has been observed. A recent value reported ${ }^{4}$ is $1.457 \AA$.

The examples mentioned here are those for which the most accurate and reliable information on molecular dimensions are available.

Molecular orbital descriptions of these molecules exhibiting the abnormally long carbon-carbon bonds have previously been made, and bond lengths predicted from calculated bond orders have been reported. In the majority of cases the Hückel approximation has been applied.

Acta Chem. Scand. 19 (1965) No. 2 
The main conclusion to be drawn from the results obtained is that the molecular orbital approaches applied cannot account for these long bonds. Invariably the predicted bond distances are too short by an amount exceeding the uncertainty of the measurements; see, e.g., Ref. 5.

In a recent paper Fischer-Hjalmars has given a thorough discussion of one of the major problems in the semi-empirical molecular orbital theory of conjugated systems, namely the problem concerned with the validity and the generality of the parameters entering the calculations. She has suggested a new, general scheme for the determination of semi-empirical parameter values in the Pariser-Parr-Pople approximation. ${ }^{6}$

In the first paper of this series ${ }^{7}$ (hereafter referred to as Paper I) this new approach was tested on some polynuclear aromatic hydrocarbons for which detailed and reliable experimental information is available. The overall agreement between predicted and observed quantities related to the ground state as well as to the electronically excited states of the molecules was satisfactory.

In the present paper the same approach will be applied in a study of the molecules phenanthrene, chrysene, and perylene. The main purpose of the investigation is to look into the possibility of describing the abnormally long bonds without introducing any modifications in the computational scheme.

In the method applied here the semi-empirical parameters entering the calculations have a functional relationship to the assumed internuclear distances in the molecules. Consequently the values of the predicted quantities may be expected to depend on the particular molecular model chosen by the evaluation of the parameter values. To investigate the sensitivity of the calculated results with respect to changes in the assumed molecular dimensions, two calculations, based on two different molecular models for perylene, have been carried out. The results obtained are discussed in Section 3.

\section{METHOD OF CALCULATION AND DETERMINATION OF PARAMETER VALUES}

A discussion of the particular procedure applied in the present study was given in Paper I.?

The numerical solution of the SCF-equations for the ground states of the molecules was evaluated on an IBM 7090 computer by means of a programme written by Mr. P. Eisenberger.

The computations of the molecular excited states where extensive configuration interaction was invoked were carried through on a UNIVAC 1107 computer. The programme used for this purpose has been written by Mr. Torbjörn Alm and kindly put at my diposal. By means of this programme, which also contains the IBM programme mentioned above, energy levels of the excited states relative to the ground state, oscillator strengths for the dipole transitions, and eigenvectors for the multiconfigurational eigenvalue problem were directly obtained from the computer. 


\section{RESULTS AND DISCUSSION}

In the present paper results obtained for the molecules phenanthrene (I), chrysene (II), and perylene (III) will be presented and discussed.

The discussion will be divided into two main parts. In 3.a an examination of some ground state properties of the molecules is given. Part 3.b is concerned with predictions made for the different electronically excited states of the molecules.

The calculations of the molecular ground states are based on a single determinant wave function, whereas configuration interaction including all the singly excited configurations has been invoked in the description of the excited singlet and triplet states.

\section{3. a. Ground state properties}

The mobile bond orders resulting from the present calculation are given in Table 1 where also corresponding values obtained by the Hückel approximation are included for comparison. For notation of bonds see Fig. 1.

Table 1. Mobile bond orders.

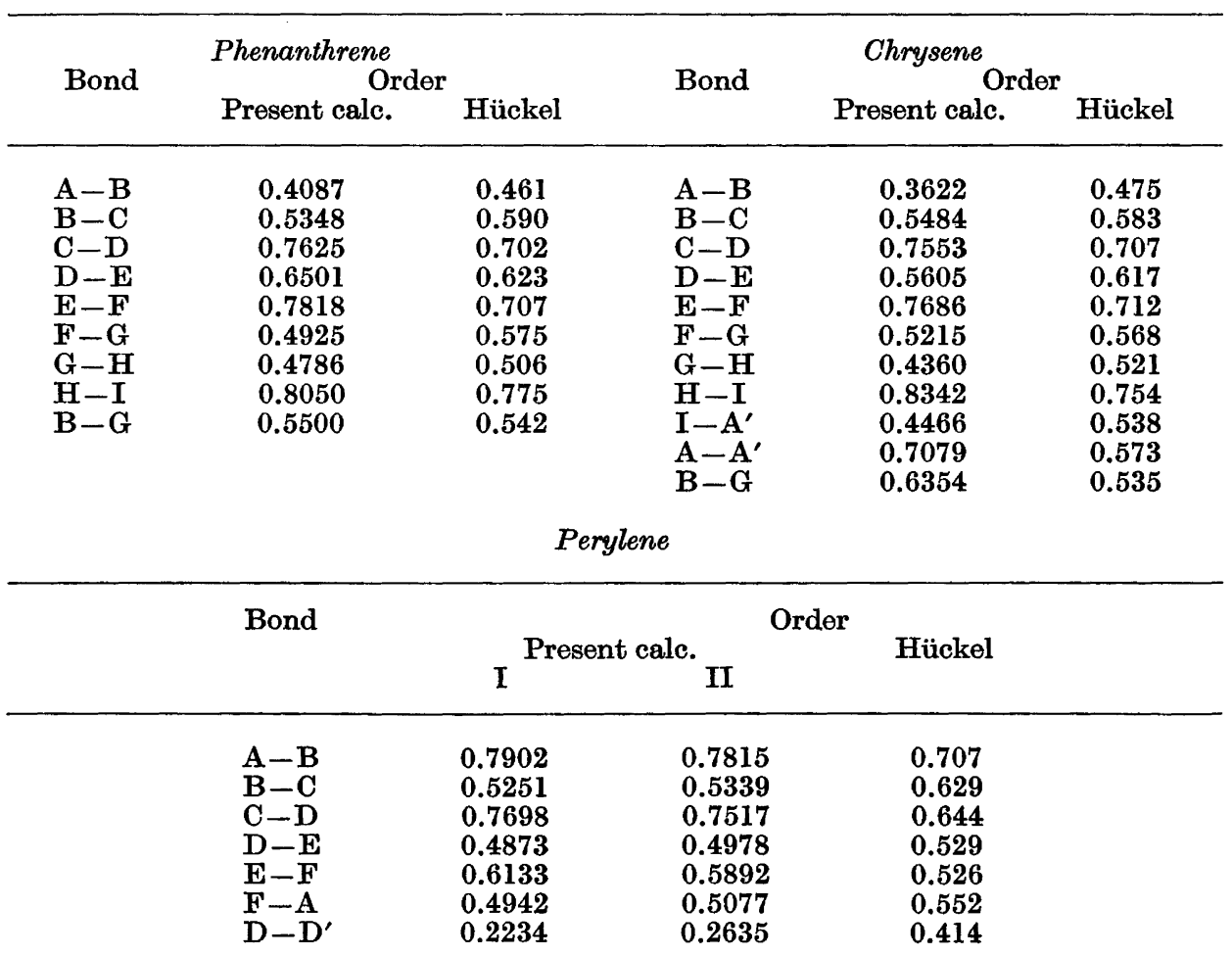

Acta Chem. Scand. 19 (1965) No. 2 

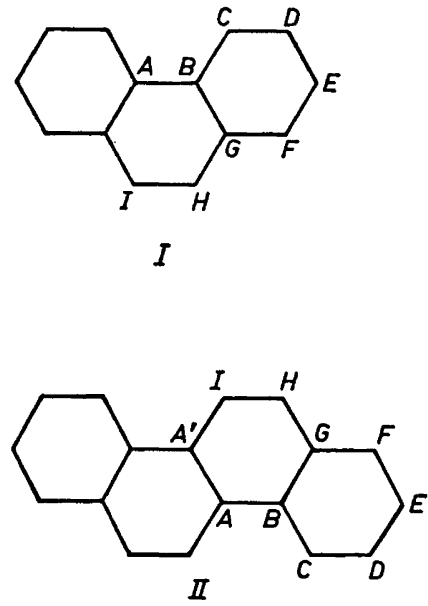

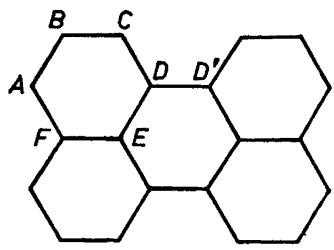

III

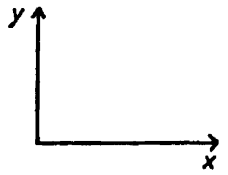

Fig. 1. Notation of bonds in phenanthrene (I), chrysene (II), and perylene (III).

The relation between bond order and bond length used here is the linear function

$$
R=1.517-0.18 p
$$

discussed and also applied in Paper I. A rather controversial point in the discussion of relationships between bond order and bond length is the assignment of a bond distance for the formal $s p^{2}-s p^{2}$ single bond. In a molecular orbital description this bond has to be considered as an extrapolation to a case where the mobile bond order is zero. The value adopted here has been suggested by Coulson, ${ }^{8}$ and is based on theoretical studies in hybridization. Dewar and Schmeising ${ }^{9}$ have suggested a value of $1.48 \AA$ for the single bond in conjugated systems. This value seems to be untenable in molecular orbital descriptions, as it would make it difficult to account for some of the observed distances in molecules such as biphenyl ${ }^{10}$ and hexaphenylbenzene. ${ }^{11}$

In Table 2 a comparison between predicted and measured bond distances is made. The estimated standard deviations (e.s.d.) of the observed values are included in parenthesis in the third column of the table.

The agreement between observed ${ }^{4}$ and predicted bond distances in phenanthrene is very good for all bonds except $F-G$ and $G-H$, the discrepancies being smaller than or equal to the reported e.s.d. values. The predicted length of the bond $F-G$ is too short by an amount twice the e.s.d., and this discrepancy therefore is hardly significant. For the bond $\mathbf{G}-\mathbf{H}$, however, the deviation between predicted and observed length is highly significant. Valence bond calculations based on Kekulé bond diagrammes also give serious discrepancies for the bonds $F-G$ and $G-H$, the differences being $+0.048 \AA$ and $-0.060 \AA$, respectively. ${ }^{4}$ Ordinary Hückel type calculations give deviations amounting to $+0.043 \AA$ and $-0.036 \AA$, respectively. ${ }^{4}$ 
Table 2. Calculated and observed bond distances ( $R$ in $\AA$ ).

\begin{tabular}{|c|c|c|c|c|c|c|}
\hline & Bond & \multicolumn{3}{|c|}{ Phenanthrene } & $\Delta \mathrm{x}$ & \\
\hline & $\begin{array}{l}\mathbf{A}-\mathbf{B} \\
\mathbf{B}-\mathbf{C} \\
\mathbf{C}-\mathbf{D} \\
\mathbf{D}-\mathbf{E} \\
\mathbf{E}-\mathbf{F} \\
\mathbf{F}-\mathbf{G} \\
\mathbf{G}-\mathbf{H} \\
\mathbf{H}-\mathbf{I} \\
\mathbf{B}-\mathbf{G}\end{array}$ & $\begin{array}{l}1.443 \\
1.421 \\
1.380 \\
1.400 \\
1.376 \\
1.428 \\
1.431 \\
1.372 \\
1.404\end{array}$ & $\begin{array}{l}1.448( \\
1.405( \\
1.383( \\
1.398( \\
1.381( \\
1.457( \\
1.390( \\
1.372( \\
1.404(\end{array}$ & $\begin{array}{l}020) \\
014) \\
014) \\
014) \\
014) \\
014) \\
014) \\
020) \\
020)\end{array}$ & $\begin{array}{l}+0.005 \\
+0.016 \\
+0.003 \\
+0.002 \\
+0.005 \\
+0.029 \\
-0.041 \\
0 \\
0\end{array}$ & \\
\hline & Bond & $R_{\text {calc. }}$ & Chrysene & & $\Delta \mathrm{x}$ & \\
\hline & $\begin{array}{l}\mathbf{A}-\mathbf{B} \\
\mathbf{B}-\mathbf{C} \\
\mathbf{C}-\mathbf{D} \\
\mathbf{D}-\mathbf{E} \\
\mathbf{E}-\mathbf{F} \\
\mathbf{F}-\mathbf{G} \\
\mathbf{G}-\mathbf{H} \\
\mathbf{H}-\mathbf{I} \\
\mathbf{I}-\mathbf{A}^{\prime} \\
\mathbf{A}-\mathbf{A}^{\prime} \\
\mathbf{B}-\mathbf{G}^{\prime}\end{array}$ & $\begin{array}{l}1.452 \\
1.418 \\
1.381 \\
1.416 \\
1.379 \\
1.423 \\
1.439 \\
1.367 \\
1.437 \\
1.390 \\
1.403\end{array}$ & $\begin{array}{l}1.468 \text { ( } \\
1.409 \\
1.381 \\
1.394( \\
1.363( \\
1.428 \\
1.421 \\
1.369 \\
1.428( \\
1.401 \text { ( } \\
1.409(\end{array}$ & $\begin{array}{l}0033) \\
0036) \\
0036) \\
0034) \\
0041) \\
0035) \\
0037) \\
0037) \\
0027) \\
0045) \\
0028)\end{array}$ & $\begin{array}{r}+0.016 \\
-0.009 \\
0 \\
-0.022 \\
+0.016 \\
+0.005 \\
-0.018 \\
+0.002 \\
+0.009 \\
+0.011 \\
+0.006\end{array}$ & \\
\hline Bond & $R_{\mathrm{I}}$ & $R_{\mathrm{II}}$ & $\begin{array}{l}\text { Perylene } \\
R_{\mathbf{X}}\end{array}$ & $\Delta \mathrm{x}_{-\mathrm{I}}$ & $\Delta \mathrm{X}_{-\mathrm{II}}$ & $\Delta_{\text {I-II }}$ \\
\hline $\begin{array}{l}\mathbf{A}-\mathbf{B} \\
\mathbf{B}-\mathbf{C} \\
\mathbf{C}-\mathbf{D} \\
\mathbf{D}-\mathbf{E} \\
\mathbf{E}-\mathbf{F} \\
\mathbf{F}-\mathbf{A} \\
\mathbf{D}-\mathbf{D}^{\prime}\end{array}$ & $\begin{array}{l}1.375 \\
1.423 \\
1.378 \\
1.429 \\
1.407 \\
1.428 \\
1.477\end{array}$ & $\begin{array}{l}1.376 \\
1.421 \\
1.383 \\
1.427 \\
1.411 \\
1.426 \\
1.470\end{array}$ & $\begin{array}{l}1.370(0.010) \\
1.418(0.008) \\
1.397(0.007) \\
1.425(0.006) \\
1.424(0.006) \\
1.400(0.008) \\
1.471(0.005)\end{array}$ & $\begin{array}{l}-0.005 \\
-0.005 \\
+0.019 \\
-0.004 \\
+0.017 \\
-0.028 \\
-0.006\end{array}$ & $\begin{array}{l}-0.006 \\
-0.003 \\
+0.014 \\
-0.002 \\
+0.013 \\
-0.026 \\
+0.001\end{array}$ & $\begin{array}{l}-0.001 \\
+0.002 \\
-0.005 \\
+0.002 \\
-0.004 \\
+0.002 \\
+0.007\end{array}$ \\
\hline
\end{tabular}

Thus by the present calculation the situation has been improved somewhat, but it appears that neither the valence bond method nor the molecular orbital approximation can account for the lengths of these two bonds.

Possible explanations of these discrepancies might be found in deviations from planarity of the molecule, and in possible in-plane deformations resulting from overcrowding of the H-atoms in ortho-positions to the bond $\mathrm{A}-\mathrm{B}$. Small, but significant distortions of the carbon skeleton from planarity have in fact been observed. ${ }^{4}$ However, it is not obvious that the effect of such a distortion should influence only the bonds $\mathbf{F}-\mathrm{G}$ and $\mathrm{G}-\mathrm{H}$.

Acta Chem. Scand. 19 (1965) No. 2 
The molecular overcrowding and its effect on the bond distances has been given detailed theoretical considerations by Coulson and Haigh. ${ }^{12}$ They conclude that in phenanthrene there should be no out of plane distortions. For the in-plane deformations they find a stretch of the bonds $A-B$ and B-C of around $0.003 \AA$ for the majority of the different van der Waals potential functions applied. Other bond stretching deformations are found to be even smaller. Thus the overcrowding of the molecule cannot explain the discrepancies found for the bonds $\mathrm{F}-\mathrm{G}$ and $\mathrm{G}-\mathrm{H}$. Before going into a closer examination of the possible effects mentioned here, a more precise experimental investigation of the molecular geometry would be necessary. In this context a comparison between the observed bond distances and valence angles in phenanthrene and chrysene is interesting. The latter molecule has been investigated in detail by Burns and Iball ${ }^{3}$ using $\mathrm{X}$-ray technique, and very accurate results are available. Chrysene may be considered as a benzophenanthrene, and the changes introduced in the bond distances of the phenanthrene part of the molecule by this addition of a phenyl group are given in Table 3. Included are also the differences between calculated values for corresponding bond distances in these two molecules. The fourth column

Table 3. Comparison between observed and calculated bond distances in phenanthrene and chrysene (in $\AA$ ).

\begin{tabular}{ccccc}
\hline Bond & Phenanthrene & Chrysene & \multicolumn{1}{c}{$\Delta_{\text {expt1. }}$} & $\Delta_{\text {calc. }}$ \\
\hline A-B & 1.448 & 1.468 & +0.020 & +0.009 \\
B-C & 1.405 & 1.409 & +0.004 & -0.003 \\
C-D & 1.383 & 1.381 & -0.002 & +0.001 \\
D-E & 1.398 & 1.394 & -0.004 & +0.016 \\
E-F & 1.381 & 1.363 & -0.018 & +0.003 \\
F-G & 1.457 & 1.428 & -0.029 & -0.005 \\
G-H & 1.390 & 1.421 & +0.031 & +0.008 \\
H-I & 1.372 & 1.369 & -0.003 & -0.005 \\
B-G & 1.404 & 1.409 & -0.005 & -0.001
\end{tabular}

of the table clearly demonstrates that except for the bonds $\mathrm{A}-\mathrm{B}, \mathrm{E}-\mathbf{F}$, $\mathrm{F}-\mathrm{G}$, and $\mathrm{G}-\mathrm{H}$ the observed distances are practically identical. The difference observed for the bond $\mathrm{A}-\mathrm{B}$ is equal to the e.s.d. of this bond in phenanthrene and therefore cannot be considered as significant. On the other hand, this difference, if real, could be partly explained by different mechanisms for releasing internal strain due to overcrowding in these two molecules. In phenanthrene the overcrowding in the region $\mathrm{A}-\mathrm{B}$ may be released by an in-plane bending maintaining the vertical plane of symmetry. In fact the observed ${ }^{4}$ value for the angle $\mathrm{ABC}$ in this molecule is $123^{\circ}$. In chrysene this type of deformation is not as easily attainable because of the equivalent overcrowded region $A^{\prime}-B^{\prime}$. According to the $X$-ray investigation ${ }^{3}$ the angle $\mathrm{ABC}$ in this molecule is increased by $2.9^{\circ}$ from its normal value $\left(120^{\circ}\right)$, whereas the increment in $\mathrm{BAI}^{\prime}$ is only $0.5^{\circ}$. Consequently bond stretching should be expected to be a more important type of deformation in chrysene than in 
phenanthrene. This is also confirmed by the results obtained for in-plane deformations by Coulson and Haigh. ${ }^{12}$ It should be added that in both cases the bond stretching is smaller than the e.s.d.'s of the experimental values.

The difference in length for the bonds $E-F$ is also close to the e.s.d. for the value reported for phenanthrene. For the bonds $F-G$ and $G-H$, however, the differences are on the limit of significance. As these two bonds are at a distance from the overcrowded regions in the molecules, it is hard to find any explanation of these discrepancies. A detailed discussion of this point requires a more refined set of experimental values for phenanthrene. A quantitative analysis of the claimed non-planarity of the carbon skeleton would be of great interest. The r.m.s. deviation between the calculated and observed distances is $0.019 \AA$, the major contribution coming from the bonds $F-G$ and $\mathrm{G}-\mathrm{H}$.

In the case of chrysene the r.m.s. difference between predicted and observed bond distances is $0.013 \AA$, the overall agreement thus being satisfactory. It is gratifying to notice that the long bond $A-B$ is fairly well accounted for.

The abnormal length of the peri-bonds in perylene and quaterrylene has been an intriguing problem. Robertson et al..$^{13}$ reported a distance of $1.50 \AA$ for these bonds in perylene, and Shrivastava and Speakman ${ }^{14}$ have shown that the average length of the peri-bonds in quaterrylene is $1.527 \AA$. The precision in the study of perylene was not very high, and a very recent reinvestigation of this molecule by Camerman and Trotter ${ }^{2}$ has shown that the peri-bonds in this molecule are $1.471 \AA$, a value that more easily can be accounted for by theoretical considerations. An electron diffraction investigation of this molecule in the vapour state recently carried out by Trætteberg ${ }^{1 *}$ does, however, support the result of Robertson et al. ${ }^{13}$ She concludes that the molecular structure of perylene may be described as two naphthalene units linked together by peri-bonds of $1.515 \AA$. Thus there is a significant discrepancy between the experimental results for the length of these bonds. This situation offers a nice opportunity to test the sensitivity of predicted molecular quantities with respect to changes in the molecular model on which the semi-empirical parameters are based. In view of this, two calculations have been carried out for perylene. In the first one the semi-empirical parameters are based on a model in accordance with the electron diffraction results, ${ }^{1}$ whereas in the second one the internuclear distances reported by Camerman and Trotter ${ }^{2}$ have been used. The corresponding sets of bond orders and bond distances obtained are labelled I and II, respectively. From the results given in Table 2 one can make the interesting conclusion that the predicted bond lengths are almost unaffected by the choice of model for the calculation. This is indicated by the figures given in the last column of the table. In this context it is particularly interesting to notice that the predicted length of the peri-bonds is in close agreement with the value reported by Camerman and Trotter irrespective of the model chosen.

* Note added in proof: Quite recently Dr. Trættebergs results on perylene have appeared in print (Proc. Roy. Soc. A 283 (1965) 557). Her final value for the length of the peri-bonds in perylene is $1.493 \AA$. 
The overall agreement between observed and predicted bond lengths is satisfactory, the r.m.s. deviations being $0.015 \AA$ and $0.013 \AA$ for sets $I$ and II, respectively. The bond $F-G$ is the only one for which the deviation between predicted and observed values is significant. It is interesting to notice that this bond is the only one in the naphthalene unit that deviates significantly from the observed bond distances in naphthalene. ${ }^{15,16}$

Another interesting result obtained by the X-ray investigation is that the molecule has a non-planar carbon skeleton due to intermolecular interactions rather than intramolecular overcrowding. ${ }^{2}$ The deformation of the molecule by too close contact between the hydrogen atoms in ortho-positions to the peri-bonds has previously been studied, and it was concluded that this overcrowding could not be made responsible for any significant distortion of the carbon framework. ${ }^{5}$

The molecular ionization potentials represent another ground state property of considerable interest. The predicted values, based on Koopmans' theorem, are presented in Table 4 where the two calculated values for perylene refer to the two different molecular models discussed above. The experimental results quoted in the table refer to different types of measurements. ${ }^{17-19}$

It is gratifying to notice that the predicted values agree rather closely with the observed ones in all the cases considered. A corresponding agreement was also obtained for the cases considered in Paper I where a discussion of this fact was given.

The table also demonstrates that for perylene the calculated value based on model II is slightly more favourable than the other one.

Table 4. Calculated and observed ionization potentials (eV).

\begin{tabular}{|l|c|c|c|c|}
\hline Molecule & $(\mathrm{IP})_{\text {calc. }}$ & $(\mathrm{IP})_{\text {obs. }}$ & Method & Reference \\
\hline \multirow{2}{*}{ Phenanthrene } & 8.06 & $\mathbf{8 . 0 3}$ & El. impact & 18 \\
& & 8.11 & Charge transfer & 17 \\
Chrysene & 7.97 & 7.71 & Charge transfer & 17 \\
Perylene & I 7.71 & 7.80 & El. negativity & 19 \\
& II 7.50 & 7.13 & Charge transfer & 17 \\
\hline
\end{tabular}

3. b. Excited states and electronictransitions

In this investigation all the singly excited configurations have been incorporated in the description of the excited states for each of the molecules studied. This has been done to compensate for the fact that the parameter scheme applied is a very general one, and not optimized for a description of electronic spectra. In addition, it was found consistent to apply configurational mixing to the same extent here as in the studies presented in Paper I.

In Tables 5, 6, and 7 predicted transition frequencies, oscillator strengths and polarizations are presented in comparison with experimental results. 
Table 5. Phenanthrene. Calculated and experimental spectrum.

\begin{tabular}{|c|c|c|c|c|c|c|}
\hline \multirow{2}{*}{$\begin{array}{l}\text { Transition from } \\
\text { gr. state to }\end{array}$} & \multicolumn{2}{|c|}{$\omega\left(\mathrm{cm}^{-1}\right)$} & \multicolumn{2}{|c|}{$f$} & \multicolumn{2}{|c|}{ polarization } \\
\hline & calc. & obs. & calc. & obs. & calc. & obs. \\
\hline 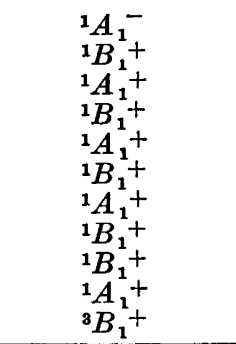 & $\begin{array}{ll}32 & 399 \\
35 & 188 \\
44 & 558 \\
47 & 552 \\
50 & 451 \\
55 & 712 \\
58 & 279 \\
58 & 666 \\
60 & 918 \\
62 & 262 \\
18 & 699\end{array}$ & $\begin{array}{ll}28300 \\
33 & 000 \\
- & \\
39 & 400 \\
47 & 100 \\
- & \\
53 & 400 \\
- & \\
\overline{56} & 400 \\
21 & 600\end{array}$ & $\begin{array}{l}0 \\
0.46 \\
0.0005 \\
1.49 \\
1.01 \\
0.18 \\
0.03 \\
0.001 \\
0.08 \\
1.20 \\
0 \\
\end{array}$ & $\begin{array}{l}0.003 \\
0.18 \\
- \\
1.09 \\
0.60 \\
- \\
0.59 \\
- \\
- \\
0.29 \\
-\end{array}$ & $\begin{array}{l}\text { forb. } \\
x \\
y \\
x \\
y \\
x \\
y \\
x \\
x \\
y \\
\text { forb. }\end{array}$ & $\begin{array}{l}y\left(L_{b}\right) \\
x\left(L_{a}\right) \\
- \\
x\left(B_{b}\right) \\
y\left(C_{b}\right) \\
- \\
y\left(B_{a}\right) \\
- \\
\bar{y}\left(\mathrm{C}_{a}\right) \\
x\left(L_{a}\right)\end{array}$ \\
\hline
\end{tabular}

The oscillator strengths have been calculated from the standard formula of Mulliken and Rieke. ${ }^{20}$

Systematic experimental studies of the electronic transitions in polynuclear aromatic hydrocarbons seem to be rather scarce. The different band systems are in most cases located with a comparatively high accuracy, but due to complications in the analysis, many of the assignments given are rather uncertain.

The experimental values for the singlet transitions in phenanthrene given in Table 5 are those reported by Klevens and Platt. ${ }^{21}$ The observed triplet level refers to a study by Evans. ${ }^{22}$

Phenanthrene belongs to the symmetry group $C_{2 v}$, and the possible symmetries for the configurations are $A_{1}$ and $B_{1}$. Transitions from the ground state to states of symmetry $A_{1}{ }^{+}$and $B_{1}{ }^{+}$are allowed. They are polarized

Table 6. Chrysene. Calculated and experimental spectrum.

\begin{tabular}{|c|c|c|c|c|c|c|}
\hline \multirow{2}{*}{$\begin{array}{l}\text { Transition from } \\
\text { gr. state to }\end{array}$} & \multicolumn{2}{|c|}{$\omega\left(\mathrm{cm}^{-1}\right)$} & \multicolumn{2}{|c|}{$f$} & \multicolumn{2}{|c|}{ polarization } \\
\hline & calc. & obs. & calc. & obs. & calc. & obs. \\
\hline $\begin{array}{l}{ }^{1} B_{u}^{-} \\
{ }^{1} B_{u}^{+} \\
{ }^{1} B_{u}^{+} \\
{ }^{1} B_{u}^{+} \\
{ }^{1} B_{u}^{+} \\
{ }^{1} B_{u}^{+} \\
{ }^{1} B_{u}^{+}\end{array}$ & $\begin{array}{ll}33 & 322 \\
34 & 366 \\
44 & 238 \\
46 & 438 \\
51 & 372 \\
59 & 085 \\
61 & 410 \\
\end{array}$ & $\begin{array}{l}27500 \\
30200 \\
37200 \\
41378 \\
45600 \\
51300 \\
54300 \\
\end{array}$ & $\begin{array}{l}0 \\
0.38 \\
1.32 \\
1.41 \\
1.01 \\
0.34 \\
0.27 \\
\end{array}$ & $\begin{array}{l}0.005 \\
0.36 \\
1.29 \\
0.4 \\
0.69 \\
0.22 \\
0.76 \\
\end{array}$ & $\begin{array}{l}\text { forb. } \\
-\quad 9.3 \\
-173.8 \\
+\quad 19.6 \\
+95.8 \\
+\quad 39.6 \\
-179.4 \\
\end{array}$ & $\begin{array}{l}L_{b} \\
L_{a} \\
B_{b} \\
\overline{C_{b}} \\
B_{a} \\
C_{a}\end{array}$ \\
\hline
\end{tabular}

Acta Chem. Scand. 19 (1965) No. 2 
Table 7. Perylene. Calculated and experimental spectrum.

\begin{tabular}{|c|c|c|c|c|c|c|}
\hline \multirow{2}{*}{$\begin{array}{l}\text { Transition from } \\
\text { gr. state to }\end{array}$} & \multicolumn{2}{|c|}{$\omega\left(\mathrm{cm}^{-1}\right)$} & \multicolumn{2}{|c|}{$f$} & \multicolumn{2}{|c|}{ polarization } \\
\hline & calc. & obs. & calc. & obs. & calc. & obs. \\
\hline $\begin{array}{l}{ }^{1} B_{3 u^{+}}+ \\
{ }^{1} B_{2 u^{+}}\end{array}$ & $\begin{array}{l}32301 \\
46771\end{array}$ & $\begin{array}{l}23050 \\
39600\end{array}$ & $\begin{array}{l}0.76 \\
1.37\end{array}$ & 0.33 & $\begin{array}{l}x \\
y\end{array}$ & $\begin{array}{l}x \\
y\end{array}$ \\
\hline $\begin{array}{l}{ }^{1} B_{3 u_{1}^{+}}+ \\
{ }^{1} B_{2 u} \\
{ }^{1} B_{3 u^{+}} \\
{ }^{1} B_{2 u}^{+} \\
{ }^{1} B_{3 u^{+}}\end{array}$ & $\begin{array}{l}47214 \\
50243 \\
54742 \\
56859 \\
59780\end{array}$ & $\begin{array}{l}40860 \\
44200 \\
-48500 \\
-\end{array}$ & $\begin{array}{l}1.20 \\
0.93 \\
0.14 \\
2.02 \\
1.07\end{array}$ & $\begin{array}{l}0.15 \\
\overline{2.17} \\
-\end{array}$ & $\begin{array}{l}x \\
y \\
x \\
y \\
x\end{array}$ & $\begin{array}{r}? \\
? \\
-\end{array}$ \\
\hline
\end{tabular}

along the $y$ - and $x$-axis respectively. The coordinate system chosen is given in Fig. 1. For definition of plus- and minus-states see, e.g., Ref. 23.

The overall agreement between predicted and observed quantities is satisfactory, and the main features of the experimental spectrum are accounted for. The calculations give systematically too high values for the transition frequencies as well as for the oscillator strengths, but the sequence of the observed transitions is nicely reproduced.

In addition to the 6 observed singlet-singlet bands, some other predicted transitions are given. First there is an allowed, but extremely weak, transition at $44558 \mathrm{~cm}^{-1}$. This band is most likely submerged in the intense observed band at $39400 \mathrm{~cm}^{-1}$. Another two allowed, but rather weak, transitions are predicted to absorb at $55712 \mathrm{~cm}^{-1}$ and $58279 \mathrm{~cm}^{-1}$, respectively. In view of the uncertainty both in calculated and observed intensities, the latter transition is related to the observed band at $53400 \mathrm{~cm}^{-1}$.

Finally an $x$-polarized, weak band is predicted at $60918 \mathrm{~cm}^{-1}$. This transition cannot be related to the observed band at $56400 \mathrm{~cm}^{-1}$ which is rather well accounted for.

The predicted symmetry of the lowest triplet state is in agreement with observations. ${ }^{24}$ The energy of this level relative to the ground state is also in accordance with recent measurements. ${ }^{22,24}$

The energy levels of the excited states of chrysene are reproduced in Table 6. Also in this case the experimental values for the singlet states are those of Klevens and Platt, ${ }^{21}$ and for one level a recent value of Becker et al. is given. ${ }^{25}$

Chrysene belongs to the symmetry group $C_{2 h}$, and the possible symmetries for the configurations are $A_{g}$ and $B_{u}$. The only allowed transitions from the ground state are those to $B_{u}^{+}$, and the corresponding transition moments have components both along the $x$ - and $y$-axis. The calculated polarizations shown in the sixth column of the table are given in terms of the angle (in degrees) between the transition moment vector and the positive $x$-axis. For coordinate system see Fig. 1. In the last column the nomenclature of Platt ${ }^{26}$ is introduced. 
On account of the large number of excited configurations in this molecule, a variety of energy levels are predicted. But only those relevant for the assignment of the observed bands are included in the table.

The first observed band at $27500 \mathrm{~cm}^{-1}$, the $L_{b}$-band, represents according to Klevens and Platt ${ }^{21}$ a short-axis polarized, very weak transition. In the present study the transition is predicted to be a forbidden one, and accordingly no direction of the transition moment can be given.

If the $\sigma_{x}$-plane is thought of as an approximate symmetry plane of the molecule, the $L_{a}$-band should be approximately parallel to this plane. The predicted angle of the transition moment vector relative to the plane is $-\mathbf{9 . 3}^{\circ}$ in nice agreement with observation. For the next, very strong $B_{b}$-band which also is long-axis polarized according to Klevens and Platt, the predicted angle is $-173.8^{\circ}$. Thus the direction of this transition also is predicted satisfactorily. In the same way the strong short-axis $C_{b}$-band is well accounted for both with respect to polarization and intensity. The same is true for the $C_{a}$-band. For the remaining $B_{a}$-band the predicted polarization bears less relation to the approximate symmetry plane. The intensity, however, is rather well accounted for.

In a recent paper Becker et al. ${ }^{25}$ have located a band of medium intensity at $41378 \mathrm{~cm}^{-1}$. From their data the $f$-value has been estimated to be around 0.4. The assignment of this band as the origin of a fourth transition has been questioned by the authors since it could fit into the vibrational progression of the strong $B_{b}$-band. As shown in Table 6 the calculations lead to an allowed, intense transition at $46438 \mathrm{~cm}^{-1}$. According to Becker et al. this transition, if real, should be polarized along the short axis of the molecule. The calculations, however, lead to a transition moment closer to the direction of the long molecular axis.

Perylene belongs to the symmetry group $D_{2 h}$, and the allowed electronic transitions from the ground state are those to states of symmetry $B_{3 u}{ }^{+}$and $B_{2 u}{ }^{+}$, respectively. The corresponding transition moments are directed along the $x$ - and $y$-axis, respectively. The coordinate system is shown in Fig. 1 .

The experimental information on the perylene spectrum is far from being complete. Extensive studies of the crystal spectrum have recently been reported, ${ }^{27-29}$ but as the interpretation of the measurements is obscured by lattice interactions leading to significant distortions of the molecular symmetry, a comparison between predictions and solution spectra is made.

The band systems of perylene in solution have been recorded by $\mathrm{Clar},{ }^{30}$ and more recently by Tanaka ${ }^{28}$ who also has given estimates of the oscillator strengths for the observed bands. According to the measurements, three strong bands at $23050 \mathrm{~cm}^{-1}, 39600 \mathrm{~cm}^{-1}$, and $48500 \mathrm{~cm}^{-1}$ have been observed. In addition a weaker band is found at $44200 \mathrm{~cm}^{-1}$. The predicted band positions are in rather poor agreement with the experimental results. This is especially true for the lowest band which is off by nearly $10000 \mathrm{~cm}^{-1}$. The discrepancy can be traced back to a very weak interaction among the configurations describing the lowest excited state of the molecule. The shift in the position of this band introduced by mixing all the singly excited configurations belonging to this symmetry, a total of 26 , is only $1550 \mathrm{~cm}^{-1}$. This, in turn, is partly due to the fact that the non-degenerate configurations

Acta Chem. Scand. 19 (1965) No. 2 
$\psi\left(i \rightarrow i^{\prime}\right)$ span this representation, and that the degenerate configurations belonging to this symmetry have comparatively high energies. On the other hand the observed band is rather broad showing substantial absorption up to around $26000 \mathrm{~cm}^{-1}$. The predictions made for the oscillator strength and the direction of the transition vector are in accordance with observations. ${ }^{28}$

In spite of the fact that the calculated transition frequencies are too high, it has been possible to make an unequivocal assignment of the observed bands.

The polarization of the second transition at $39600 \mathrm{~cm}^{-1}$ has not been definitely established, but arguments in favour of a short-axis polarized transition have been given. ${ }^{28}$ The present calculations lead to the result that there are two close lying transitions of different polarizations in this region. The most intense one is polarized along the $y$-axis in accordance with the suggestion of Tanaka.

The third, weak band at $44200 \mathrm{~cm}^{-1}$ is related to a short-axis polarized transition, the calculated frequency of which is too high by about $6000 \mathrm{~cm}^{-1}$. In view of the comparatively good agreement between calculated and observed $f$-values, this assignment should be rather certain.

The most intense band in the observed spectrum is rather well accounted for by a predicted $y$-polarized transition which according to the calculations has the highest intensity.

Another two transitions are predicted. First there should be a very weak, but-allowed transition absorbing in a region between the weak band at $44200 \mathrm{~cm}^{-1}$ and the intense band at $48500 \mathrm{~cm}^{-1}$. This transition will most likely be submerged in the latter, and therefore difficult to locate. Rather close to the most intense band, at a higher frequency a long-axis polarized transition of medium intensity is predicted. This band has not been observed, but a rather broad shoulder on the peak of the observed ${ }^{28} 48500 \mathrm{~cm}^{-1}$ band indicates the presence of such a transition, which should be polarized along the long axis of the molecule.

Acknowledgements. The author wishes to express his gratitude to Professor I. FischerHjalmars for her stimulating and inspiring guidance of this work. Thanks are also due to Mr. T. Alm for placing his SCF CI programme at the author's disposal, and for his kind assistance in the use of the programme. Finally the author should like to thank Dr. M. Trætteberg, Trondheim, Norway, for communicating her unpublished results on perylene.

This investigation has been supported by Statens Naturvetenskapliga Forskningsråd.

\section{REFERENCES}

1. Trætteberg, M. Private communication.

2. Camerman, A. and Trotter, J. Proc. Roy. Soc. A 279 (1964) 129.

3. Burns, D. M. and Iball, J. Proc. Roy. Soc. A 257 (1960) 491.

4. Trotter, J. Acta Cryst. 16 (1963) 605.

5. Coulson, C. A. and Skancke, P. N. J. Chem. Soc. 19622775.

6. Fischer-Hjalmars, I. In Löwdin, P.-O. and Pullman, B. Molecular Orbitals in Chemistry, Physics and Biology, Academic Press 1964.

7. Skancke, P. N. Acta Chem. Scand. 18 (1964) 1671.

8. Coulson, C. A. Victor Henri Memorial Volume, Desoer, Liége 1948, p. 15.

9. Dewar, M. J. S. and Schmeising, H. N. Tetrahedron 5 (1959) 166. 
10. Almenningen, A. and Bastiansen, O. Kgl. Norske Videnskab. Selskabs, Skrifter 1958 No. 3.

11. Almenningen, A., Bastiansen, O. and Skancke, P. N. Acta Chem. Scand. 12 (1958) 1215.

12. Coulson, C. A. and Haigh, C. W. Tetrahedron 19 (1963) 527.

13. Donaldson, D. M., Robertson, M. J. and White, J. G. Proc. Roy. Soc. A 220 (1953) 311.

14. Shrivastava, H. N. and Speakman, J. C. Proc. Roy. Soc. A 257 (1960) 477.

15. Almenningen, A., Bastiansen, O. and Dyvik, F. Acta Cryst. 14 (1961) 1056.

16. Cruickshank, D. W. J. and Sparks, R. A. Proc. Roy. Soc. A 258 (1960) 270.

17. Kuroda, H. Nature 201 (1964) 1214.

18. Wacks, M. E. and Dibeler, V. H. J. Chem. Phys. 31 (1959) 1557.

19. Becker, R. S. and Wentworth, W. E. J. Am. Chem. Soc. 85 (1963) 2210.

20. Mulliken, R. S. and Rieke, C. A. Rept. Progr. Phys. 8 (1941) 231.

21. Klevens, H. B. and Platt, J. R. J. Chem. Phys. 17 (1949) 470.

22. Evans, D. F. J. Chem. Soc. 19571351.

23. Pariser, R. J. Chem. Phys. 24 (1956) 250.

24. MeClure, D. J. Chem. Phys. 17 (1949) 905.

25. Becker, R. S., Singh, I. S. and Jackson, E. A. J. Chem. Phys. 38 (1963) 2144.

26. Platt, J. R. J. Chem. Phys. 17 (1949) 484.

27. Hochstrasser, R. M. Can. J. Chem. 39 (1961) 451.

28. Tanaka, J. Bull. Chem. Soc. Japan 36 (1963) 1237.

29. Hohstrasser, R. M. J. Chem. Phys. 40 (1964) 2559.

30. Clar, E. Spectrochim. Acta 4 (1950) 119.

Received November 19, 1964. 